\title{
Haematological and Biochemical Reference Values for Healthy Population of Maferinyah Rural Community in Guinea
}

\author{
Abdoul Habib Béavogui, ${ }^{1,2}$ Almamy Amara Touré ${ }^{1},{ }^{1}$ Daouda Camara, ${ }^{1}$ \\ Abdoulaye Doumbouya, ${ }^{1}$ Malick Minkael Sylla, ${ }^{1}$ Sékou Touré, ${ }^{3}$ Ahmadou Hamidou Togo, ${ }^{3}$ \\ Mamadou Saliou Diallo, ${ }^{1}$ Alexandre Delamou, ${ }^{1,4,5}$ Issaka Sagara, ${ }^{3}$ Alassane Dicko, ${ }^{3}$ \\ and Abdoulaye Djimdé ${ }^{3}$ \\ ${ }^{1}$ Centre National de Formation et de Recherche en Santé Rurale de Maferinyah, Forecariah, Guinea \\ ${ }^{2}$ Bioclinical and Fundamental Sciences Chair, Department of Medical Sciences, Faculty of Health Science and Techniques, \\ Gamal Abdel Nasser University of Conakry, Conakry, Guinea \\ ${ }^{3}$ Malaria Research and Training Center (MRTC), Département d'Epidémiologie des Affections Parasitaires (DEAP), Faculté de \\ Médecine de Pharmacie et d'Odonto-Stomatologie (FMPOS), Université des Sciences, \\ des Techniques et des Technologies de Bamako (USTTB), Mali \\ ${ }^{4}$ Department of Public Health, Faculty of Health Sciences and Techniques, Gamal Abdel Nasser University, Conakry, Guinea \\ ${ }^{5}$ Centre d'Excellence Africain pour la Prévention et le Contrôle des Maladies Transmissibles (CEA-PCMT), Gamal Abdel \\ Nasser University, Conakry, Guinea \\ Correspondence should be addressed to Almamy Amara Touré; almamy@maferinyah.org
}

Received 25 April 2020; Accepted 17 July 2020; Published 3 August 2020

Academic Editor: Giuseppe Piccione

Copyright ( 92020 Abdoul Habib Béavogui et al. This is an open access article distributed under the Creative Commons Attribution License, which permits unrestricted use, distribution, and reproduction in any medium, provided the original work is properly cited.

\begin{abstract}
Guinea's reference ranges for biological parameters rely on those of Caucasian values. Variability in reference ranges according to the context is well-documented. We conducted this study for the purpose of future malaria clinical trials that assess the efficacy and safety of malaria drugs. A repeated cross-sectional study was carried out, in an apparently healthy cohort population. Surveys took place in Maferinyah rural community, which is located at $75 \mathrm{~km}$ from the capital. The $2.5^{\text {th }}$ and $97.5^{\text {th }}$ percentiles were determined nonparametrically and stood for reference intervals. Reference values were determined separately for males and females according to ranges of age (6-10 years of age; 11-15 years of age; 16-45 years of age). Differences between genders were tested using the MannWhitney test, while the Friedman test was performed to test differences within each gender group according to the seasons. A total of 450 volunteers were enrolled. The median age was 13. Males 16-45 years of age had significantly higher hematologic and biochemical values compared to a female of the same age (for hematological parameters: Mean Cell Hemoglobin Concentration MCHC $p \leq 0.001$, Platelets $p \leq 0.001$, monocytes $p=0.0305$, eosinophils $p=0.0225$; for biochemical parameters: Aspartate aminotransferase AST $p \leq 0.001$, Alanine Aminotransferase ALT $p \leq 0.001$, creatinine $p \leq 0.001$ ). We noticed significant seasonal variations for all the biochemical parameters and some hematologic parameters (Mean Corpuscular Hemoglobin $\mathrm{MCH}, \mathrm{MCHC}$, Mean Cell volume). This is the first study establishing hematologic and biochemical parameters in Guinea. These findings provide a useful guide for the clinical researchers and care providers. Studies on large scale and in different settings would be also desirable.
\end{abstract}

\section{Introduction}

Patient management relies heavily on the speed of clinical laboratory results, upon which medical diagnosis is estab- lished $[1,2]$. Health care professional needs to draw a conclusion based on laboratory tests results, which depends on preanalytical and analytical factors [3-5]. These factors may influence laboratory results and may have an impact on 
hematological and biochemical tests' results. For instance, physiological factors that may influence laboratory results include age, activity, bed rest, food ingestion, alcohol ingestion, menstrual cycle, obesity, oral contraceptives, posture, pregnancy, race, gender, smoking, and time of day [4]. Variations of reference values due to physiological and demographical factors such as gender, age, regions, are welldocumented [6-12]. Hence, the provision of reliable reference intervals remains an important task for clinical laboratories and diagnostic test manufacturers [13]. As a result, an expert committee in the US has recommended that each manufacturer of diagnostic kit must provide reference intervals [14]. Laboratories have also been instructed to validate the reference ranges and prove that they are applicable for their environment and populations [14].

In most developing countries, including Guinea, laboratory reference values in use are from Caucasian data. However, these developing countries do not have the same physiological and environmental patterns as industrialized countries. Their health conditions and the distribution of healthy populations are totally different from that of developed countries. The Mafèrinyah training and research center in Guinea is planning to conduct a phase-III clinical trial to assess antimalarial drug safety. This requires the use of local population reference values for Alanine aminotransferase (ALAT), Aspartate aminotransferase (ASAT), bilirubin, creatinine, and blood count in order to follow biological safety of these new agents. Unfortunately, there were no such reference values to define participants' enrollment criteria. Our study aimed at establishing reference values of haematological and biochemical parameters in healthy population in Mafèrinyah subprefecture.

\section{Methodology}

2.1. Study Design, Site, and Population. This was a crosssectional study with a cohort participant. Three surveys were carried out between March 2011 and December 2013; the first one in the middle of the dry season (March), the second one at the start of the rainy season (May), and the last one at the end of the rainy season (November). Subprefecture of Mafèrinyah is part of the Prefecture of Forécariah. The population of the subprefecture of Mafèrinyah was estimated at 38,934 inhabitants and distributed between 6 villages as follows: Mafèrinyah centre: 14,595 inhabitants; Madinagbé: 8,321 inhabitants; Moribayah: 4,951 inhabitants; Fandié: 3,636 inhabitants; Koket: 3,023 inhabitants; and Maléah: 2,086 inhabitants. Mafèrinyah centre, which hosts the health Centre (CS) and the Centre for Training and Research in Rural Health (CFRSR), is located at $75 \mathrm{~km}$ from the capital Conakry and halfway $(25 \mathrm{~km})$ between the prefectures of Coyah and Forécariah (Unpublished data: Monitoring of health centre activities 2006). Participants in this study were from the Maferinyah community and aged 6-45 years old. A total of 450 volunteers were enrolled. Inclusion criteria included those who lived in the area for at least 6 months, nonpregnant healthy women, apparently healthy clinically, willingness to participate to the study without a long-term travel. Patients with confirmed malaria and those who do not want to participate to the study were excluded. The sample size was computed according to the Clinical and Laboratory Standards Institute (CLSI) guidelines for the establishment of reference interval [13], which recommends a minimum of 120 participants in each category used for stratification, we therefore completed this size to 150 participants in each category to take into account the loss of participants.

2.2. Survey Collection Procedure. Data were collected at Mafèrinyah Centre using a standardized individual questionnaire developed according to the objectives of the study. All participants meeting the inclusion criteria were enrolled. Data on general information, complete clinical examination, and biological examination were performed. Malaria diagnostic was performed for all participants. That information was transcribed into the case report forms by study clinical investigators. Clinical examination was carried out by the physician of the research team in order to make sure that all participants met the inclusion criteria.

2.3. Ethical Considerations. Ethical approval was obtained from the National Ethics Committee of Guinea called "Comité National de l'Ethique de la Recherche en Santé de Guinée" (Protocol number: 07/CNERS/10). Written informed consent was obtained from all participants after the purpose of the study was fully explained. For participants under 18, parents or guardians signed for them. For participants unable to read the French language, the content of the informed consent form was translated into the local language in the presence of an independent witness.

\subsection{Laboratory Analysis}

2.4.1. Blood Collection. About $5 \mathrm{ml}$ of venous blood were collected in an ethylene diamine tetra acetic acid (EDTA) vacutainer tube for haematology and in a serum separator vacutainer tube for biochemistry. All samples were kept at laboratory temperature and away from heat and sunlight.

2.4.2. Hematologic Analysis. Haematological analyses were performed by using validated ABX Pentra 60 Hematology Analyzers (Horiba-ABX, Montpellier, France). Reagents, calibrators, and controls were provided by the manufacturer from the kit. Controls were run daily and calibrators each time a new batch of controls arrived. Samples analyses were performed within 4 hours after blood collection, and the results were printed for each participant and kept in his/her case report form (CRF).

2.4.3. Biochemical Analysis. Biochemical analyses were performed by using Piccolo ${ }^{\circledR}$ xpress $^{\mathrm{TM}}$ Chemistry Analyzer (USA). Test tubes, Reagents, calibrators, and controls were provided by Elitech Diagnostics (Sees, France). Blood samples for biochemical analyses were centrifuged, and serum was collected and analyzed within 4 hours, and the results were printed for each participant and kept in his/her CRF.

2.4.4. Pregnancy Test. A pregnancy test was offered to female participants who were at least 13 years old prior to their enrollment. We used the method of urine strip, and all positive cases were excluded. 
TABLE 1: Hematological and biochemical reference values of participants, for 6-10 years of age $(R 1+R 2+R 3)^{\mathfrak{E}}$.

\begin{tabular}{|c|c|c|c|c|c|c|c|}
\hline \multirow{2}{*}{ Parameters } & \multicolumn{3}{|c|}{ Female } & \multicolumn{3}{|c|}{ Male } & \multirow{2}{*}{$p$ value" } \\
\hline & $N$ & Median & Reference values* & $N$ & Median & Reference values & \\
\hline WBC $\left(1000 / \mathrm{mm}^{3}\right)$ & 208 & 7.00 & $4.30-12.92$ & 235 & 7.60 & $4.59-14.35$ & 0.0278 \\
\hline $\mathrm{RBC}\left(10^{\wedge} 6 \mathrm{~mm}^{3}\right)$ & 208 & 4.49 & $3.53-5.38$ & 235 & 4.48 & $3.36-5.46$ & 0.8788 \\
\hline Hemoglobin (g/dl) & 208 & 11.10 & $8.59-13.00$ & 235 & 11.10 & $8.09-12.72$ & 0.0852 \\
\hline Hematocrit (\%) & 208 & 35.10 & $25.789-41.00$ & 235 & 34.60 & 25.87-39.55 & 0.2309 \\
\hline $\operatorname{MCV}\left(\mu \mathrm{mm}^{3}\right)$ & 208 & 78.00 & $62.85-89.00$ & 235 & 77.00 & $62.70-88.00$ & 0.0428 \\
\hline $\mathrm{MCH}(\mathrm{pg})$ & 208 & 25.20 & $19.64-28.88$ & 235 & 24.80 & $18.81-28.20$ & 0.4986 \\
\hline $\mathrm{MCHC}(\mathrm{g} / \mathrm{dl})$ & 208 & 32.10 & $28.90-34.70$ & 235 & 32.30 & $28.79-34.81$ & 0.2688 \\
\hline Platelet $\left(1000 / \mathrm{mm}^{3}\right)$ & 208 & 289.00 & $91.60-457.15$ & 235 & 301.00 & $39.85-458.70$ & 0.2688 \\
\hline $\operatorname{MPV}\left(\mu \mathrm{m}^{3}\right)$ & 208 & 7.90 & $6.70-10.06$ & 235 & 8.10 & $6.50-9.73$ & 0.8566 \\
\hline Lymphocytes (\%) & 208 & 50.30 & $31.41-63.43$ & 235 & 45.90 & $28.47-61.45$ & $\leq 0.001$ \\
\hline Monocytes (\%) & 208 & 9.90 & $6.623-16.78$ & 235 & 10.20 & $6.79-18.02$ & 0.2581 \\
\hline Neutrophils (\%) & 208 & 34.10 & $20.20-52.95$ & 235 & 35.90 & $21.44-57.65$ & 0.1342 \\
\hline Eosinophils (\%) & 208 & 3.20 & $1.10-15.55$ & 235 & 4.40 & $1.20-25.26$ & $\leq 0.001$ \\
\hline Basophils (\%) & 208 & 0.80 & $0.40-2.62$ & 235 & 0.70 & $0.40-1.81$ & $\leq 0.001$ \\
\hline AST (UI/L) & 208 & 29.80 & $12.99-51.24$ & 235 & 32.00 & $14.65-59.32$ & 0.0057 \\
\hline $\operatorname{ALT}(\mathrm{UI} / \mathrm{L})$ & 208 & 19.00 & $9.10-37.63$ & 235 & 19.45 & $9.10-54.65$ & 0.3069 \\
\hline $\mathrm{T} / \mathrm{Bil}(\mathrm{mg} / \mathrm{L})$ & 208 & 0.50 & $0.20-1.42$ & 235 & 0.60 & $0.20-2.12$ & 0.0074 \\
\hline Creatinine $(\mathrm{mg} / \mathrm{L})$ & 208 & 0.54 & $0.24-0.73$ & 235 & 0.53 & $0.30-0.81$ & 0.7588 \\
\hline
\end{tabular}

${ }^{\mathfrak{E}} R 1$ : first round, $R 2$ : second round, $R 3$ : third round. ${ }^{*}$ References values stand for $2.5^{\text {th }}-97.5^{\text {th }}$ percentiles. WBC: white blood cell, RBC: red blood cell, MCV: mean cell volume, $\mathrm{MCH}$ : mean corpuscular hemoglobin, MCHC: mean cell hemoglobin concentration, MPV: mean platelet volume, ALT: alanine aminotransferase, AST: aspartate aminotransferase, T/Bil: total bilirubin. " $p$ value based on Mann-Whitney test.

2.4.5. Quality Control. Controls were run on the instrument daily according to the manufacturer recommendations. No analysis was performed if any control was out of range. In addition, an internal monitoring system was implemented to assess all the laboratory processes. Moreover, Mafèrinyah's research laboratory was subjected to external quality assessments for haematology and biochemistry by the Malaria Research and Training Centre of Bamako (Mali). The laboratory also complied with the principles of Good Clinical and Laboratory Practice (GCP/GLP) $[15,16]$.

2.5. Data Management and Statistical Analysis. Data were double-entered into an Access database and checked visually. The $2.5^{\text {th }}$ and $97.5^{\text {th }}$ percentiles were determined nonparametrically and stood for reference intervals [13]. Outliers within each subgroup were identified using the Dixon method [17]. To sum up, the extreme values were retained in the distribution if $\mathrm{D} / \mathrm{R}, 0.33$, where $D$ is the absolute difference between the most extreme distribution and the next value and $R$ is the Range (maximum-minimum). Reference values were determined separately for males and females according to the range of age (6-10 years of age; 11-15 years of age; 16-45 years of age). Differences between genders were tested using the MannWhitney test, while the Friedman test was performed to test differences within each gender group according to seasons. For the multiple comparison of groups that show the difference in variances Nemenyi was used. Data analysis was carried out using R 3.6.2. All tests were considered significant with $p<0.05$.

\section{Results}

A total of 450 participants 6-45 years of age were enrolled. The median age was 13 , the majority of participants were students (71\%); $53 \%$ of study population were females; the ethnic group Soussou accounted for $65 \%$ of study sample (additional file). In participants of 6-10 years of age, males had significantly higher White Blood Cell (WBC), eosinophils, Aspartate Aminotransferase (AST), and total bilirubin (T/Bil) values compared to females (Table 1). However, Mean Cell Volume (MCV), lymphocytes, and basophils values were significantly higher in female participants than in males (Table 1). In the subgroup of 11-15 years of age, males had significantly higher hemoglobin, hematocrit, eosinophils, basophils, AST, ALT, T/Bil, and creatinine than in female (Table 2), female MCV, Mean Corpuscular Hemoglobin $(\mathrm{MCH})$ values were significantly higher than in males (Table 2). In the subgroup 16-45 years of age, males had significantly higher values of red blood cells, hemoglobin, hematocrit, Mean Cell Hemoglobin Concentration (MCHC), Platelets, monocytes, eosinophils, AST, ALT (Alanine aminotransferase), and creatinine compared to female (Table 3); but at the same time, WBC, MPV, and neutrophils were significantly higher in female participants compared to those in male (Table 3). Reference values of females 6-10 years of age varied significantly between three (3) rounds for the following parameters: hemoglobin, hematocrit, MCV, AST, ALT, TBIL, and creatinine (Table 4). In a pairwise analysis, the following parameters, hematocrit, T/Bil, and creatinine, showed a significant difference between the first and the second round on one hand and the first and 
TABLE 2: Hematological and biochemical reference values of participants, $11-15$ years of age $(R 1+R 2+R 3)^{\mathfrak{E}}$.

\begin{tabular}{|c|c|c|c|c|c|c|c|}
\hline \multirow{2}{*}{ Parameters } & \multicolumn{3}{|c|}{ Female } & \multicolumn{3}{|c|}{ Male } & \multirow{2}{*}{${ }^{*} p$ value } \\
\hline & $N$ & Median & Reference values & $N$ & Median & Reference values & \\
\hline WBC $\left(1000 / \mathrm{mm}^{3}\right)$ & 209 & 6.60 & $3.8-11.5$ & 150 & 6.40 & $4.09-10.80$ & 0.5199 \\
\hline $\mathrm{RBC}\left(10^{\wedge} 6 \mathrm{~mm}^{3}\right)$ & 209 & 4.56 & $3.82-5.49$ & 150 & 4.57 & $3.75-5.81$ & 0.9868 \\
\hline Hemoglobin (g/dl) & 209 & 11.80 & $9.50-13.73$ & 150 & 11.50 & $9.00-14.05$ & 0.0027 \\
\hline Hematocrit (\%) & 209 & 37.20 & $28.64-43.80$ & 150 & 36.20 & $29.34-45.28$ & 0.0086 \\
\hline $\operatorname{MCV}\left(\mu \mathrm{mm}^{3}\right)$ & 209 & 82.00 & $67.85-93.00$ & 150 & 81.00 & $65.85-90.00$ & 0.0012 \\
\hline $\mathrm{MCH}(\mathrm{pg})$ & 209 & 26.00 & $20.07-30.90$ & 150 & 25.30 & $19.57-29.10$ & 0.0141 \\
\hline $\mathrm{MCHC}(\mathrm{g} / \mathrm{dl})$ & 209 & 31.70 & $29.085-35.20$ & 150 & 31.30 & $28.89-34.70$ & 0.2229 \\
\hline Platelet $\left(1000 / \mathrm{mm}^{3}\right)$ & 209 & 272.00 & $94.70-441.65$ & 150 & 260.00 & $110.25-409.25$ & 0.3470 \\
\hline $\operatorname{MPV}\left(\mu \mathrm{m}^{3}\right)$ & 209 & 8.30 & $6.90-10.03$ & 150 & 8.10 & $6.67-10.00$ & 0.0068 \\
\hline Lymphocytes (\%) & 209 & 46.50 & $29.51-61.72$ & 150 & 44.70 & $30.495-59.10$ & 0.1745 \\
\hline Monocytes (\%) & 209 & 10.50 & $6.470-16.21$ & 150 & 10.20 & $6.34-18.10$ & 0.9745 \\
\hline Neutrophils (\%) & 209 & 36.20 & $20.70-56.11$ & 150 & 35.40 & $19.89-52.82$ & 0.1544 \\
\hline Eosinophils (\%) & 209 & 3.60 & $1.10-20.25$ & 150 & 6.10 & $1.49-18.62$ & $\leq 0.001$ \\
\hline Basophils (\%) & 209 & 0.60 & $0.40-1.00$ & 150 & 0.70 & $0.39-5.41$ & 0.0015 \\
\hline AST (UI/L) & 209 & 27.00 & $10.66-40.00$ & 150 & 31.30 & $13.31-52.00$ & $\leq 0.001$ \\
\hline $\operatorname{ALT}(\mathrm{UI} / \mathrm{L})$ & 209 & 17.00 & $9.10-28.30$ & 150 & 19.00 & $9.10-40.747$ & 0.0202 \\
\hline $\mathrm{T} / \mathrm{Bil}(\mathrm{mg} / \mathrm{L})$ & 209 & 0.50 & $0.20-1.12$ & 150 & 0.50 & $0.20-1.32$ & $\leq 0.001$ \\
\hline Creatinine $(\mathrm{mg} / \mathrm{L})$ & 209 & 0.62 & $0.29-0.92$ & 150 & 0.70 & $0.39-1.00$ & 0.0046 \\
\hline
\end{tabular}

${ }^{*} p$ value based on Mann-Whitney test. ${ }^{\mathfrak{E}}(R 1$ : first round; $R 2$ : second round; $R 3$ : third round).

the third round on other hand (additional file). Conversely, ALT yielded a significant difference between the 1 st and third round on one hand and between 2 nd and third round on the other hand. MCV only shows a significant change between March and November while AST shows a significant difference between all the rounds (additional file). Reference values of females 11-15 years of age varied significantly between the three rounds for the following parameters: WBC, hematocrit, $\mathrm{MCH}$, MCHC, MPV, AST, monocytes, AST, ALT, and T/Bil (Table 5). Pairwise analysis indicated significant WBC and $\mathrm{MCH}$ variation between the 1 st and the 2 nd round, between 2 nd and the $3^{\text {rd }}$ round; we noticed the same result for T/Bil (additional file). Conversely, Hematocrit varied significantly between March and May on one side, and between March and November on the other side; MPV changed significantly between March and November (additional file). MCHC, AST, and ALT varied significantly between all rounds (additional file). Reference values of females from 16 to 45 years varied significantly between the three rounds for the following parameters: CCMH, AST, ALT, and TBILIRUBINE (Table 6). We observed significant changes in MCHC value between March and May and between March and November, but no significant variation between May and November (additional file). ALT and AST varied significantly between all rounds whereas T/Bil varied only between March and May and between March and November (additional file).

Reference values of males 6-10 years of age varied significantly between the three rounds for the following parameters: RBC, Hematocrit, MCV, MCH, MCHC, Neutrophils, Basophils, AST, ALT, T/Bil, and Creatinine
(Table 7). In pairwise analysis, $\mathrm{RBC}, \mathrm{MCH}$, and neutrophils varied significantly between March and November. Hematocrit value changed significantly between March and May, and between March and November. We noticed significant variation in MCV values between March and May, and between May and November. $\mathrm{CMCH}$, basophils/Bil, and creatinine changed significantly between March and May, and between March and November. ALT values changed significantly between March and November, and between May and November. We also observed significant variation in AST values between all seasons. Reference values of males 11-15 years of age varied significantly between the three rounds for the following parameters: Hematocrit, MCH, MCHC, AST, ALT, and T/Bil (Table 8). In addition, Hematocrit, MCHC, and T/Bil show significant variation between March and May, and between March and November; MCH varied significantly between May and November. AST and ALT values changed significantly between seasons. Reference values of males from 16 to 45 years of age varied significantly between the three rounds for the following parameters: Hematocrit, $\mathrm{MCH}, \mathrm{MCHC}, \mathrm{MPV}$, monocytes, Neutrophils, AST, ALT, and T/Bil (Table 9). We noticed significant variation in hematocrit, $\mathrm{MCH}$, and $\mathrm{MCHC}$ between March and May, and between March and November. MPV ad neutrophils values only vary significantly between March and November. Conversely, monocytes change significantly between March and May, and between May and November. We observed significant variation in ALT and T/Bil values between March and November, and between May and November. AST changed significantly between all rounds. 
TABLE 3: Hematological and biochemical reference values of participants, $16-45$ years of age $(R 1+R 2+R 3)^{£}$.

\begin{tabular}{|c|c|c|c|c|c|c|c|}
\hline \multirow{2}{*}{ Parameters } & \multicolumn{3}{|c|}{ Female } & \multicolumn{3}{|c|}{ Male } & \multirow{2}{*}{${ }^{*} p$ value } \\
\hline & $N$ & Median & Reference values & $N$ & Median & Reference values & \\
\hline WBC $\left(1000 / \mathrm{mm}^{3}\right)$ & 206 & 6.40 & $4.00-10.43$ & 208 & 6.00 & $3.90-10.62$ & 0.0408 \\
\hline $\mathrm{RBC}\left(10^{\wedge} 6 \mathrm{~mm}^{3}\right)$ & 206 & 4.41 & $3.63-5.71$ & 208 & 5.04 & $4.23-6.10$ & $\leq 0.001$ \\
\hline Hemoglobin (g/dl) & 206 & 11.60 & $8.83-13.60$ & 208 & 13.40 & $8.20-15.50$ & $\leq 0.001$ \\
\hline Hematocrit (\%) & 206 & 36.70 & $28.64-43.80$ & 208 & 41.90 & $29.74-48.16$ & $\leq 0.001$ \\
\hline $\operatorname{MCV}\left(\mu \mathrm{mm}^{3}\right)$ & 206 & 83.00 & $63.85-97.00$ & 208 & 83.00 & $62.70-95.15$ & 0.7061 \\
\hline $\mathrm{MCH}(\mathrm{pg})$ & 206 & 26.40 & $19.35-31.22$ & 208 & 26.80 & $18.50-31.35$ & 0.1172 \\
\hline $\mathrm{MCHC}(\mathrm{g} / \mathrm{dl})$ & 206 & 26.50 & $28.38-34.40$ & 208 & 32.30 & 29.04-34.82 & $\leq 0.001$ \\
\hline Platelet $\left(1000 / \mathrm{mm}^{3}\right)$ & 206 & 201.20 & $86.1-413.6$ & 208 & 226.00 & $54.95-526.15$ & $\leq 0.001$ \\
\hline $\operatorname{MPV}\left(\mu \mathrm{m}^{3}\right)$ & 206 & 8.40 & $7.00-10.12$ & 208 & 8.30 & $6.80-10.10$ & $\leq 0.001$ \\
\hline Lymphocytes (\%) & 206 & 43.90 & $27.30-62.28$ & 208 & 45.00 & $26.00-63.40$ & 0.2118 \\
\hline Monocytes (\%) & 206 & 9.30 & $5.40-15.56$ & 208 & 9.90 & $6.99-17.29$ & 0.0305 \\
\hline Neutrophils (\%) & 206 & 39.60 & $22.71-60.93$ & 208 & 35.50 & $20.57-60.03$ & 0.0088 \\
\hline Eosinophils (\%) & 206 & 3.90 & $0.99-20.04$ & 208 & 5.20 & $1.10-22.64$ & 0.0225 \\
\hline Basophils (\%) & 206 & 0.60 & $0.39-1.43$ & 208 & 0.60 & $0.40-1.30$ & 0.0068 \\
\hline AST (UI/L) & 206 & 25.00 & $10.37-44.69$ & 208 & 28.60 & $13.56-49.15$ & $\leq 0.001$ \\
\hline $\operatorname{ALT}(\mathrm{UI} / \mathrm{L})$ & 206 & 18.00 & $9.10-47.14$ & 208 & 20.00 & $9.10-46.09$ & 0.0042 \\
\hline T/Bil (mg/L) & 206 & 0.50 & $0.20-1.20$ & 208 & 0.60 & $0.20-2.22$ & 0.7418 \\
\hline Creatinine $(\mathrm{mg} / \mathrm{L})$ & 206 & 0.76 & $0.30-1.03$ & 208 & 0.97 & $0.29-1.31$ & $\leq 0.001$ \\
\hline
\end{tabular}

* $p$ value based on Mann-Whitney test. ${ }^{\mathfrak{E}}$ ( $R 1$ : first round; $R 2$ : second round; $R 3$ : third round).

TABle 4: Reference values among Female cohort 6-10 years of age who attended all rounds (1 to 3). $N=49$.

\begin{tabular}{|c|c|c|c|c|c|c|c|}
\hline \multirow[t]{2}{*}{ Parameters (unit) } & \multicolumn{2}{|c|}{ 1st round (dry season) } & \multicolumn{2}{|c|}{ 2nd round (rainy season) } & \multicolumn{2}{|c|}{$\begin{array}{c}\text { 3rd round (end of rainy } \\
\text { season) }\end{array}$} & \multirow[t]{2}{*}{${ }^{*} p$ value } \\
\hline & Median & References value & Median & References value & Median & References value & \\
\hline WBC $\left(1000 / \mathrm{mm}^{3}\right)$ & 8.10 & $4.50-16.23$ & 7.60 & $4.55-12.80$ & 7.60 & $4.85-12.32$ & 0.2596 \\
\hline $\mathrm{RBC}\left(10^{\wedge} 6 \mathrm{~mm}^{3}\right)$ & 4.56 & $3.44-5.71$ & 4.50 & $3.88-5.37$ & 4.33 & $3.25-5.20$ & 0.0797 \\
\hline Hemoglobin $(\mathrm{g} / \mathrm{dl})$ & 11.20 & $7.83-12.19$ & 11.10 & $9.60-12.70$ & 11.10 & $8.04-13.15$ & 0.0137 \\
\hline Hematocrit (\%) & 36.80 & $26.62-40.34$ & 33.10 & $28.60-37.70$ & 33.90 & $25.65-39.35$ & 0.0011 \\
\hline $\operatorname{MCV}\left(\mu \mathrm{mm}^{3}\right)$ & 78.00 & $15.88-88.00$ & 74.00 & $59.50-85.50$ & 79.00 & 67-85 & 0.0946 \\
\hline $\mathrm{MCH}(\mathrm{pg})$ & 23.75 & $19.81-27.34$ & 24.80 & $19.55-28.70$ & 25.80 & $21.23-28.21$ & 0.0074 \\
\hline $\mathrm{MCHC}(\mathrm{g} / \mathrm{dl})$ & 30.40 & 28.71-31.95 & 33.40 & $31.50-35.10$ & 32.70 & $30.50-34.77$ & $\leq 0.001$ \\
\hline Platelet $\left(1000 / \mathrm{mm}^{3}\right)$ & 305.00 & $60.88-445.62$ & 288.00 & $88.20-467.50$ & 304.00 & $151.25-476.75$ & 0.5523 \\
\hline $\operatorname{MPV}\left(\mu \mathrm{m}^{3}\right)$ & 8.20 & $6.91-9.29$ & 8.20 & $6.80-9.80$ & 7.90 & 6.8-9.7 & 0.0196 \\
\hline Lymphocytes (\%) & 47.60 & 28.94-61.96 & 46.40 & $27.25-60.15$ & 43.70 & $31.06-62.38$ & 0.1301 \\
\hline Monocytes (\%) & 10.45 & $6.83-15.64$ & 9.80 & $6.95-15.30$ & 10.50 & $6.48-19.09$ & 0.1919 \\
\hline Neutrophils (\%) & 34.10 & $21.66-53.11$ & 35.90 & $22.20-58.95$ & 36.30 & $21.67-67.38$ & 0.348 \\
\hline Eosinophils (\%) & 4.40 & $0.85-20.76$ & 4.70 & $1.20-20.70$ & 4.20 & $1.03-26.76$ & 0.4444 \\
\hline Basophils (\%) & 0.75 & $0.41-3.64$ & 0.60 & $0.35-0.90$ & 0.70 & $0.45-1.05$ & 0.0761 \\
\hline AST (UI/L) & 37.00 & $29.00-55.75$ & 31.70 & $21.15-57.45$ & 23.10 & $13.08-57.24$ & $\leq 0.001$ \\
\hline ALT (UI/L) & 22.00 & $16.12-50.25$ & 20.90 & $10.81-59.84$ & 10.65 & $9.10-35.85$ & $\leq 0.001$ \\
\hline $\mathrm{T} / \mathrm{Bil}(\mathrm{mg} / \mathrm{L})$ & 0.60 & $0.40-2.54$ & 0.30 & $0.20-0.85$ & 0.40 & $0.20-1.17$ & $\leq 0.001$ \\
\hline Creatinine $(\mathrm{mg} / \mathrm{L})$ & 0.50 & $0.30-0.70$ & 0.58 & $0.40-0.69$ & 0.56 & $0.39-0.77$ & 0.6065 \\
\hline
\end{tabular}

${ }^{*} p$ value based on Friedman test.

\section{Discussion}

Establishing the ranges of value is a key step toward clinical trials. Clinical and routine laboratories use Caucasian values to make decision in Guinea. However, socioeconomic and environmental (seasons) conditions may influence hematologic and biochemical parameters. It appears the need for having its own values as recommended by 
TABLe 5: Reference values among Female cohort 11-15 years of age who attended all rounds (1 to 3). $N=40$.

\begin{tabular}{|c|c|c|c|c|c|c|c|}
\hline \multirow[t]{2}{*}{ Parameters (unit) } & \multicolumn{2}{|c|}{ 1st round (dry season) } & \multicolumn{2}{|c|}{2 nd round (rainy season) } & \multicolumn{2}{|c|}{$\begin{array}{c}\text { 3rd round (end of rainy } \\
\text { season) }\end{array}$} & \multirow[t]{2}{*}{${ }^{*} p$ value } \\
\hline & Median & References value & Median & References value & Median & References value & \\
\hline WBC $\left(1000 / \mathrm{mm}^{3}\right)$ & 6.60 & $4.19-8.56$ & 7.30 & $4.9-11.9$ & 5.85 & $4.30-12.52$ & 0.0019 \\
\hline $\mathrm{RBC}\left(10^{\wedge} 6 \mathrm{~mm}^{3}\right)$ & 4.54 & $4.07-5.86$ & 4.45 & $3.83-5.26$ & 4.36 & $3.23-5.21$ & 0.0811 \\
\hline Hemoglobin (g/dl) & 11.50 & $10.19-13.90$ & 11.90 & $10.2-13.5$ & 11.35 & $8.61-13.20$ & 0.0067 \\
\hline Hematocrit (\%) & 37.95 & $34.05-45.04$ & 35.90 & $30.7-40.4$ & 34.40 & 27.01-40.59 & 0.0654 \\
\hline $\operatorname{MCV}\left(\mu \mathrm{mm}^{3}\right)$ & 85.00 & $71.90-95.03$ & 79.00 & 64-92 & 81.00 & $70.00-88.97$ & 0.0125 \\
\hline $\mathrm{MCH}(\mathrm{pg})$ & 25.50 & $21.25-36.06$ & 26.60 & $20.8-30.9$ & 26.45 & $22.20-30.28$ & $\leq 0.001$ \\
\hline $\mathrm{MCHC}(\mathrm{g} / \mathrm{dl})$ & 30.60 & $28.80-303.07$ & 33.50 & $32.3-35.2$ & 32.80 & $31.4-34.3$ & 0.9092 \\
\hline Platelet $\left(1000 / \mathrm{mm}^{3}\right)$ & 263.00 & $189.83-389.52$ & 276.00 & $22-386$ & 271.50 & $83.60-599.23$ & 0.0196 \\
\hline $\operatorname{MPV}\left(\mu \mathrm{m}^{3}\right)$ & 8.25 & 7.29-9.90 & 8.40 & $7.0-10.2$ & 7.90 & 6.4-9.2 & 0.2899 \\
\hline Lymphocytes (\%) & 47.60 & $29.43-76.63$ & 46.50 & $30.1-58.0$ & 48.10 & 26.46-59.95 & 0.0011 \\
\hline Monocytes (\%) & 10.65 & $7.08-16.81$ & 9.40 & $6.5-12.4$ & 10.45 & $5.81-16.09$ & 0.1637 \\
\hline Neutrophils (\%) & 32.55 & $20.68-54.23$ & 38.40 & $23.1-56.7$ & 36.85 & $21.01-62.44$ & 0.3907 \\
\hline Eosinophils (\%) & 4.05 & $1.10-16.45$ & 3.20 & $1.2-21.5$ & 3.20 & $0.91-21.47$ & 0.1948 \\
\hline Basophils (\%) & 0.60 & $0.4-1.0$ & 0.70 & $0.3-1.0$ & 0.60 & $0.3-0.9$ & $\leq 0.001$ \\
\hline AST (UI/L) & 30.00 & $22.97-34.02$ & 24.30 & $12.3-38.4$ & 17.10 & $9.82-27.20$ & $\leq 0.001$ \\
\hline $\operatorname{ALT}(\mathrm{UI} / \mathrm{L})$ & 20.00 & $12.95-28.05$ & 13.60 & $9.10-27.55$ & 9.10 & $9.10-14.57$ & $\leq 0.001$ \\
\hline T/Bil (UI/L) & 0.65 & $0.50-1.12$ & 0.40 & $0.2-0.9$ & 0.30 & $0.2-0.9$ & $\leq 0.001$ \\
\hline Creatinine $(\mathrm{mg} / \mathrm{L})$ & 0.60 & $0.4-0.8$ & 0.64 & $0.2-0.9$ & 0.66 & $0.18-0.86$ & 0.5514 \\
\hline
\end{tabular}

$* p$ value based on Friedman test.

TABle 6: Reference values among Female cohort for 16-45 years of age who attended all rounds (1 to 3 ). $N=32$.

\begin{tabular}{|c|c|c|c|c|c|c|c|}
\hline \multirow[t]{2}{*}{ Parameters (unit) } & \multicolumn{2}{|c|}{ 1st round (dry season) } & \multicolumn{2}{|c|}{ 2nd round (rainy season) } & \multicolumn{2}{|c|}{$\begin{array}{l}\text { 3rd round (end of rainy } \\
\text { season) }\end{array}$} & \multirow[t]{2}{*}{$* p$ value } \\
\hline & Median & References value & Median & References value & Median & References value & \\
\hline $\mathrm{WBC}\left(1000 / \mathrm{mm}^{3}\right)$ & 6.40 & $4.03-8.85$ & 6.90 & $4.45-11.80$ & 6.30 & $4.21-8.78$ & 0.1615 \\
\hline $\mathrm{RBC}\left(10^{\wedge} 6 \mathrm{~mm}^{3}\right)$ & 4.46 & $3.62-5.71$ & 4.41 & $3.67-5.69$ & 4.38 & $3.41-5.27$ & 0.773 \\
\hline Hemoglobin (g/dl) & 11.40 & $7.82-13.62$ & 11.90 & $9.22-14.00$ & 11.70 & $8.40-13.356$ & 0.1679 \\
\hline Hematocrit (\%) & 37.60 & $26.87-44.17$ & 35.50 & $28.85-41.73$ & 35.85 & $27.68-40.46$ & 0.0829 \\
\hline $\operatorname{MCV}\left(\mu \mathrm{mm}^{3}\right)$ & 83.50 & $62.95-95.45$ & 81.00 & $63.75-93.75$ & 80.00 & $66.90-94.10$ & 0.5783 \\
\hline $\mathrm{MCH}(\mathrm{pg})$ & 25.35 & $16.48-82.35$ & 27.30 & $20.30-32.65$ & 26.45 & 20.23-30.95 & 0.0976 \\
\hline $\mathrm{MCHC}(\mathrm{g} / \mathrm{dl})$ & 30.35 & $28.18-31.69$ & 33.10 & $28.00-35.55$ & 32.70 & $30.12-33.80$ & $\leq 0.001$ \\
\hline Platelet $\left(1000 / \mathrm{mm}^{3}\right)$ & 236.00 & $119.65-407.70$ & 258.00 & $138-406$ & 265.00 & $125.60-447.07$ & 0.1203 \\
\hline $\operatorname{MPV}\left(\mu \mathrm{m}^{3}\right)$ & 8.35 & 7.46-9.55 & 8.40 & $7.05-10.07$ & 8.60 & 7.04-10.33 & 0.3585 \\
\hline Lymphocytes (\%) & 41.60 & $31.19-63.55$ & 41.30 & $28.43-60.50$ & 44.70 & $26.39-58.72$ & 0.3607 \\
\hline Monocytes (\%) & 9.85 & $5.38-14.73$ & 9.20 & $5.73-15.73$ & 8.90 & $5.37-14.69$ & 0.6763 \\
\hline Neutrophils (\%) & 38.45 & $21.28-54.22$ & 41.90 & $22.62-58.62$ & 38.65 & $24.53-61.95$ & 0.327 \\
\hline Eosinophils (\%) & 5.85 & $1.41-20.54$ & 3.30 & $0.82-17.62$ & 2.90 & $0.87-21.20$ & 0.0528 \\
\hline Basophils (\%) & 0.60 & $0.38-7.22$ & 0.60 & $0.32-1.05$ & 0.60 & $0.40-0.98$ & 0.4169 \\
\hline AST (UI/L) & 28.50 & $22.33-40.67$ & 26.40 & $15.05-43.30$ & 19.40 & $9.20-30.99$ & $\leq 0.001$ \\
\hline ALT (UI/L) & 19.00 & $13.77-50.60$ & 14.35 & $9.10-37.31$ & 10.05 & $9.1-27.3$ & $\leq 0.001$ \\
\hline T/Bil (UI/L) & 0.60 & $0.40-0.82$ & 0.50 & $0.40-0.82$ & 0.30 & $0.20-1.11$ & $\leq 0.001$ \\
\hline Creatinine (mg/L) & 0.70 & $0.30-0.92$ & 0.73 & $0.30-0.92$ & 0.78 & $0.36-1.02$ & 0.0992 \\
\hline
\end{tabular}

${ }^{*} p$ value based on Friedman test. 
TABLE 7: Reference values among Male cohort 6-10 years of age who attended all rounds (1 to 3 ). $N=46$.

\begin{tabular}{|c|c|c|c|c|c|c|c|}
\hline \multirow{2}{*}{ Parameters (unit) } & \multicolumn{2}{|c|}{ 1st round (dry season) } & \multicolumn{2}{|c|}{ 2nd round (rainy season) } & \multicolumn{2}{|c|}{$\begin{array}{l}\text { 3rd round (end of rainy } \\
\text { season) }\end{array}$} & \multirow[t]{2}{*}{$* p$ value } \\
\hline & Median & References value & Median & References value & Median & References value & \\
\hline WBC $\left(1000 / \mathrm{mm}^{3}\right)$ & 8.10 & $4.50-16.23$ & 7.60 & $4.55-12.80$ & 7.60 & $4.85-12.32$ & 0.2596 \\
\hline $\mathrm{RBC}\left(10^{\wedge} 6 \mathrm{~mm}^{3}\right)$ & 4.56 & $3.44-5.71$ & 4.50 & $3.88-5.37$ & 4.33 & $3.25-5.20$ & 0.7266 \\
\hline Hemoglobin (g/dl) & 11.20 & $7.83-12.19$ & 11.10 & $9.6-12.7$ & 11.10 & $8.04-13.15$ & 0.0096 \\
\hline Hematocrit (\%) & 36.80 & $26.62-40.34$ & 33.10 & $28.6-37.7$ & 33.90 & $25.65-39.35$ & $\leq 0.001$ \\
\hline $\operatorname{MCV}\left(\mu \mathrm{mm}^{3}\right)$ & 78.00 & $15.88-88.00$ & 74.00 & $59.5-85.5$ & 79.00 & 67-85 & 0.0046 \\
\hline $\mathrm{MCH}(\mathrm{pg})$ & 23.75 & $19.81-27.34$ & 24.80 & $19.55-28.70$ & 25.80 & $21.23-28.21$ & 0.0022 \\
\hline $\mathrm{MCHC}(\mathrm{g} / \mathrm{dl})$ & 30.40 & 28.71-31.95 & 33.40 & $31.5-35.1$ & 32.70 & $30.50-34.77$ & $\leq 0.001$ \\
\hline Platelet $\left(1000 / \mathrm{mm}^{3}\right)$ & 305.00 & $60.88-445.62$ & 288.00 & $88.2-467.5$ & 304.00 & $151.25-476.75$ & 0.3735 \\
\hline $\operatorname{MPV}\left(\mu \mathrm{m}^{3}\right)$ & 8.20 & 6.91-9.29 & 8.20 & $6.80-9.80$ & 7.90 & $6.80-9.70$ & 0.8298 \\
\hline Lymphocytes (\%) & 47.60 & 28.94-61.96 & 46.40 & $27.25-60.15$ & 43.70 & $31.06-62.38$ & 0.4553 \\
\hline Monocytes (\%) & 10.45 & $6.83-15.64$ & 9.80 & $6.95-15.30$ & 10.50 & $6.48-19.09$ & 0.2364 \\
\hline Neutrophils (\%) & 34.10 & $21.66-53.11$ & 35.90 & $22.20-58.95$ & 36.30 & $21.67-67.38$ & 0.05803 \\
\hline Eosinophils (\%) & 4.40 & $0.85-20.76$ & 4.70 & $1.2-20.7$ & 4.20 & $1.03-26.76$ & 0.5881 \\
\hline Basophils (\%) & 0.75 & $0.41-3.64$ & 0.60 & $0.35-0.90$ & 0.70 & $0.45-1.05$ & $\leq 0.001$ \\
\hline AST (UI/L) & 37.00 & $29.00-55.75$ & 31.70 & $21.15-57.45$ & 23.10 & $13.08-57.24$ & $\leq 0.001$ \\
\hline ALT (UI/L) & 22.00 & $16.12-50.25$ & 20.90 & $10.81-59.84$ & 10.65 & $9.10-35.85$ & $\leq 0.001$ \\
\hline T/Bil (UI/L) & 0.60 & $0.40-2.54$ & 0.30 & $0.20-0.85$ & 0.40 & $0.20-1.17$ & $\leq 0.001$ \\
\hline Creatinine $(\mathrm{mg} / \mathrm{L})$ & 0.50 & $0.3-0.7$ & 0.58 & $0.40-0.69$ & 0.56 & $0.39-0.77$ & $\leq 0.001$ \\
\hline
\end{tabular}

${ }^{*} p$ value based on Friedman test.

TABLE 8: Reference values among Male cohort 11-15 years of age who attended all rounds (1 to 3). $N=32$.

\begin{tabular}{|c|c|c|c|c|c|c|c|}
\hline \multirow[t]{2}{*}{ Parameters (unit) } & \multicolumn{2}{|c|}{$1^{\text {st }}$ round (dry season) } & \multicolumn{2}{|c|}{$2^{\text {nd }}$ round (rainy season) } & \multicolumn{2}{|c|}{$\begin{array}{l}3^{\text {rd }} \text { round (end of rainy } \\
\text { season) }\end{array}$} & \multirow[t]{2}{*}{$* p$ value } \\
\hline & Median & References value & Median & References value & Median & References value & \\
\hline $\mathrm{WBC}\left(1000 / \mathrm{mm}^{3}\right)$ & 6.55 & $4.28-10.69$ & 6.50 & $3.96-10.25$ & 5.90 & $4.90-7.76$ & 0.3563 \\
\hline $\mathrm{RBC}\left(10^{\wedge} 6 \mathrm{~mm}^{3}\right)$ & 4.59 & $4.14-5.61$ & 4.50 & $3.92-5.28$ & 4.46 & $3.16-5.46$ & 0.1184 \\
\hline Hemoglobin (g/dl) & 11.65 & $9.91-13.60$ & 11.80 & $9.96-12.94$ & 11.30 & $7.32-14.18$ & 0.0011 \\
\hline Hematocrit (\%) & 38.10 & $32.60-44.46$ & 35.60 & $30.49-37.45$ & 34.40 & $24.64-42.28$ & 0.0617 \\
\hline $\operatorname{MCV}\left(\mu \mathrm{mm}^{3}\right)$ & 82.00 & $71.88-87.00$ & 79.00 & $66.5-86.0$ & 78.00 & $62.6-87.8$ & 0.0946 \\
\hline $\mathrm{MCH}(\mathrm{pg})$ & 25.00 & $20.90-26.95$ & 26.20 & $20.98-29.05$ & 25.80 & $19.04-29.14$ & 0.0074 \\
\hline $\mathrm{MCHC}(\mathrm{g} / \mathrm{dl})$ & 30.60 & $29.46-31.62$ & 33.70 & $31.45-34.99$ & 32.60 & $30.12-34.76$ & $\leq 0.001$ \\
\hline Platelet $\left(1000 / \mathrm{mm}^{3}\right)$ & 252.50 & $180.40-389.57$ & 267.50 & $121.00-347.75$ & 278.00 & $179.0-347.4$ & 0.5523 \\
\hline $\operatorname{MPV}\left(\mu \mathrm{m}^{3}\right)$ & 8.20 & 7.15-9.58 & 8.15 & 7.01-9.56 & 8.20 & $6.88-9.42$ & 0.9447 \\
\hline Lymphocytes (\%) & 44.60 & $30.98-51.37$ & 48.45 & $33.86-59.48$ & 47.30 & $32.10-58.16$ & 0.1301 \\
\hline Monocytes (\%) & 10.20 & $6.27-21.07$ & 9.75 & $5.21-13.93$ & 10.80 & $7.74-18.24$ & 0.1919 \\
\hline Neutrophils (\%) & 35.75 & $26.27-51.35$ & 33.10 & $22.05-50.11$ & 35.90 & $20.24-51.30$ & 0.348 \\
\hline Eosinophils (\%) & 6.30 & $1.54-15.99$ & 6.60 & $2.21-27.10$ & 6.10 & $1.28-13.62$ & 0.4444 \\
\hline Basophils (\%) & 0.70 & $0.38-2.03$ & 0.60 & $0.36-0.84$ & 0.60 & $0.40-1.18$ & 0.0761 \\
\hline AST (UI/L) & 34.50 & $26.32-53.80$ & 29.00 & $15.03-45.15$ & 22.40 & $12.92-34.68$ & $\leq 0.001$ \\
\hline ALT (UI/L) & 22.50 & $15.32-41.12$ & 19.14 & $9.88-32.65$ & 12.02 & $9.10-20.25$ & $\leq 0.001$ \\
\hline T/Bil (mg/L) & 0.60 & $0.48-0.82$ & 0.30 & $0.20-0.94$ & 0.30 & $0.20-5.42$ & $\leq 0.001$ \\
\hline Creatinine (mg/L) & 0.70 & $0.48-0.90$ & 0.68 & $0.24-0.92$ & 0.74 & $0.50-0.97$ & 0.6065 \\
\hline
\end{tabular}

${ }^{*} p$ value based on Friedman test.

the Experts [14]. To the best of our knowledge, this is the first study ever done in Guinea, in order to address the malaria clinical trials' need, which must rely on context-based values. Hence, take into seasonal variations are more realistic since malaria is seasonal dependent. Our study highlighted an important variability in 
TABLE 9: Reference values for Male cohort 16-45 years of age who attended all rounds (1 to 3). $N=32$.

\begin{tabular}{|c|c|c|c|c|c|c|c|c|}
\hline \multirow[t]{2}{*}{ Parameters (unit) } & \multicolumn{2}{|c|}{$1^{\text {st }}$ round (dry season) } & \multicolumn{3}{|c|}{$2^{\text {nd }}$ round (rainy season) } & \multicolumn{2}{|c|}{$\begin{array}{c}3^{\text {rd }} \text { round (end of rainy } \\
\text { season) }\end{array}$} & \multirow[t]{2}{*}{$p$ value } \\
\hline & Median & References value & Median & References value & $\mathrm{N}$ & Median & References value & \\
\hline WBC $\left(1000 / \mathrm{mm}^{3}\right)$ & 6.40 & $4.11-11.04$ & 6.25 & $3.14-9.97$ & & 6.30 & $4.21-8.78$ & 0.346 \\
\hline $\mathrm{RBC}\left(10^{\wedge} 6 \mathrm{~mm}^{3}\right)$ & 5.11 & $4.37-6.08$ & 4.98 & $4.17-6.26$ & & 4.38 & $4.21-8.78$ & 0.0651 \\
\hline Hemoglobin (g/dl) & 13.40 & $10.41-14.85$ & 13.85 & $10.62-15.50$ & & 11.70 & $3.41-5.27$ & 0.755 \\
\hline Hematocrit (\%) & 44.60 & $34.95-49.45$ & 40.80 & $31.02-46.14$ & & 35.85 & $8.40-13.36$ & $\leq 0.001$ \\
\hline $\operatorname{MCV}\left(\mu \mathrm{mm}^{3}\right)$ & 84.00 & $68.60-95.00$ & 83.00 & $67.25-91.88$ & & 80.00 & $66.9-94.1$ & 0.3171 \\
\hline $\mathrm{MCH}(\mathrm{pg})$ & 25.80 & $20.32-29.23$ & 27.15 & $21.31-31.14$ & & 26.45 & $20.23-30.95$ & 0.0015 \\
\hline MCHC (g/dl) & 30.50 & 29.24-31.06 & 33.20 & $31.95-34.84$ & & 32.70 & $30.12-33.80$ & $\leq 0.001$ \\
\hline Platelet $\left(1000 / \mathrm{mm}^{3}\right)$ & 222.00 & $101.50-414.80$ & 209.00 & $50.00-735.12$ & & 265.00 & $125.60-447.07$ & 0.9382 \\
\hline $\operatorname{MPV}\left(\mu \mathrm{m}^{3}\right)$ & 8.70 & $7.28-10.03$ & 8.50 & 6.81-9.59 & & 8.60 & 7.04-10.33 & 0.0207 \\
\hline Lymphocytes (\%) & 44.70 & $26.68-65.61$ & 47.05 & $27.18-61.14$ & & 44.70 & $26.39-58.72$ & 0.3526 \\
\hline Monocytes (\%) & 9.90 & $7.34-16.05$ & 9.25 & $6.54-13.46$ & & 8.90 & $5.37-14.69$ & 0.0116 \\
\hline Neutrophils (\%) & 33.60 & $19.56-62.17$ & 36.15 & $20.86-61.11$ & & 38.65 & $24.53-61.95$ & 0.0274 \\
\hline Eosinophils (\%) & 5.40 & $1.17-20.73$ & 6.05 & $1.21-18.86$ & & 2.90 & $0.87-21.20$ & 0.2758 \\
\hline Basophils (\%) & 0.70 & $0.40-2.73$ & 0.60 & $0.31-1.08$ & & 0.60 & $0.40-0.98$ & 0.0668 \\
\hline AST (UI/L) & 33.00 & $24.4-76.0$ & 26.65 & $14.97-43.75$ & & 19.40 & $9.20-30.99$ & $\leq 0.001$ \\
\hline ALT (UI/L) & 20.00 & $14.7-43.3$ & 19.11 & $9.34-42.13$ & & 10.05 & $9.1-27.3$ & $\leq 0.001$ \\
\hline T/Bil (UI/L) & 0.70 & $0.50-0.96$ & 0.60 & $0.21-3.83$ & & 0.30 & $0.20-1.11$ & $\leq 0.001$ \\
\hline Creatinine (mg/L) & 1.00 & $0.27-1.33$ & 0.96 & $0.39-1.21$ & & 0.78 & $0.36-1.02$ & 0.0715 \\
\hline
\end{tabular}

* $p$ value based on Friedman test.

hematologic and biochemical values that will serve as well as routine and clinical laboratories.

On the overall rounds $(R 1+R 2+R 3)$, males had the highest levels of hemoglobin, hematocrit, basophils, AST, ALT, T/Bil (total bilirubin), and creatinine. Males have usually higher hematologic and biochemical counts than female, because of hormonal influence like androgen [18, 19]; according to Daniel the increase in size and volume of muscle fibers is an associated increase in the number of red blood cells, and, therefore rise in hematocrit and hemoglobin values [19]. In addition to that, menstrual period may lead down women RBC count. Our findings comply with several studies [10, 12, 20-24]. Conversely, females had higher levels of $\mathrm{MCV}$ and $\mathrm{MCH}$ and involved those of 6-15 years of age. Similarly, we hypothesize that WBC elevation in female adults compared to male ones is due to multiples aggressions (infectious diseases, injuries) that men are exposed in that context. Those findings are in accordance with the previous reports $[9,25]$. We also found a decrease of WBC levels as age increases, which is in line with previous studies $[7,26]$. In brief, we noticed few differences between females and males of 6-10 years of age, in fact, only 7 out of 18 parameters were significant. However, variations are more remarkable in males and females 11-15 years of age and 16-45 years of age with, respectively, 11 out of 18 parameters and 14 out of 18 parameters significant. This suggests that hematologic parameters change a little in early life regardless of age and gender [27]. We noticed few changes in female 6-10 years of age parameters, only 6 out of 18 parameters varied over time. Hematologic and biochemical parameters remained higher at the dry season compared to rainy seasons. The study period might explain that result, in fact, the two last surveys took place after 2 years of the first one, because of logistical reasons. Another relevant reason is the possible impact of malaria, even participants were careful to screen, and there might be some subclinical and submicroscopic cases. As females grew up the parameters change significantly, among 11-15 years of age cohort, 10 out 18 parameters had varied significantly through the rounds. We have already discussed about the influence of the age on parameter levels [7]. Females experienced various modifications such as the hormonal effect that lead to the menstrual period. Hematocrit, ALT, AST, and T/Bil decreased over time while $\mathrm{MCH}, \mathrm{MCHC}$, and MPV increased over time. The WBC and monocytes fluctuate through all the seasons. Interestingly, we found few parameter changes in female cohort adult, only 4 out of 18 parameters varied over time. $\mathrm{MCH}$ fluctuated trough the seasons, whereas AST, ALT, and T/Bil have decreased over time. On the contrary to females of the same age, most of the 6-10 years of age males' parameters changed through seasons. So, 12 out of 18 parameters varied according to seasons. Hematocrit, basophils, AST, ALT, $\mathrm{T} / \mathrm{Bil}$, and creatinine decreased significantly between dry season and end of the rainy season, while neutrophils, $\mathrm{MCH}$, and MCHC increased significantly over time. As we were working on different times of day, this may change some parameters count. Diurnal variation of the neutrophils count has been established with the highest levels in the afternoon and lowest levels in the morning at rest [1]. In addition, as we have previously seen before, AST remained the only parameter that changed between all rounds.

Males of 11-15 years of age experienced few hematologic and biochemical parameters changes compared to females of the same age. We found 7 out of 18 parameters that varied 
through the periods. As we have seen before, Hematocrit and Biochemicals parameters (AST, ALT, and T/Bil) decreased significantly between the three rounds while $\mathrm{MCH}, \mathrm{MCHC}$ increased significantly over time. AST and ALT values changed significantly through all the seasons. Many studies pointed out the impact of seasons on biochemical and haemotogical parameters; these include environmental factors (temperature, exposure to sunlight, climate, etc.), individual's factors (exercise, physical activity, diet, etc.) [28-34]. Of note that is, the rainy season is the favorable time in diseases increase like malaria and other parasitic diseases not screen in this study. Besides, other characteristics not studied in this study might explain the seasonal variations. For instance, one could seek to know if the harvest period influences the nutritional state and subsequently the parameter values. Therefore, specific study design to seasonal variations would be interesting.

Cohort adult males $16-45$ years of age had slightly different changes in hematologic and biochemical parameters compared to females of the same age. We noticed 9 out of 18 parameters significantly varied over time. So, hematocrit, MPV, monocytes, AST, ALT, T/Bil, and Creatinine decreased significantly between seasons while $\mathrm{MCH}, \mathrm{MCHC}$, and Neutrophils raised over time. We failed to know if participants were nonsmokers or smokers, because we were only relying on participants' statements. It has been reported that cigarette smokers have higher average leukocyte counts than nonsmokers [1]. The increase is greatest (about 30\%) in heavy smokers who inhale and affects neutrophils, lymphocytes, and monocytes [1]. We also notice again AST change between all rounds. Our study has limitations and strengths, we did not perform all the analysis that are needed to rule out diseases among volunteers like HIV, hepatitis C, syphilis, sickle cell disease, thalassemia, and micronutrient deficiencies. To fill this gap, we performed careful clinical evaluations through well-trained physicians. Only some parameters that we needed for malaria clinical trial assessment were included. Stratification in ranges of age according to gender has also affected the sample size. We consider that other studies on hematologic and biochemical parameters that will include more parameters, and diverse participants from different regions will reinforce evidences. In the meantime, these findings remain useful in the scarcity of data. Hence, we recommend its use by clinical laboratories.

\section{Conclusion}

Our study reveals interesting patterns of hematologic and biochemical parameters in Guinea. Little change in parameters was observed in males and females aged 6-15 years old. However, in accordance with previous reports, males' adults had higher parameters than females. We noticed parameter variations through the seasons mostly for few hematologic parameters (MCH, MCHC, and MCV) and for all the biochemical tests (ALT, AST, creatinine, and T/Bil). AST remained the only parameter that has changed through all the seasons. These results provide a useful guide for the clinical researchers and care providers.

\section{Data Availability}

The datasets in csv Excel format and the software R scripts used to support the findings of this study are available from the corresponding author upon request.

\section{Conflicts of Interest}

The authors state that there is no conflict with the parties cited in this study.

\section{Authors' Contributions}

Abdoul Habib Beavogui wrote the first draft; Almamy Amara Touré and Alexandre Delamou performed analysis and interpretation of the data, Abdoulaye Doumbouya, Daouda Camara, Malick Minkael, Sekou Touré, Ahmadou Hamidou Togo, and Mamadou Saliou Diallo reviewed the first draft; Alhassance Dicko, Issaka Sagara, and Abdoulaye Djimdé designed the protocol of the study and provided analysis plan. All the authors read and approved the final manuscript.

\section{Acknowledgments}

The authors are grateful to the Malaria Research Centre of Bamako for their technical support, also the volunteers who took part in this study. We also thank our following colleagues for the data collection: Mohamed Yattara, Gnépou Camara, Amadou Sow, Nèma Goumou, Pascal Millimounou, Mamadou Saliou Touré. This study was backed by the European \& Developing Countries Clinical Trials Partnership (EDCTP).

\section{Supplementary Materials}

The supplementary material includes the Pairwise Analysis (Supplementary Materials)

\section{References}

[1] R. A. McPherson and M. R. Pincus, Henry's Clinical Diagnosis and Management by Laboratory Methods, ELSEVIER SAUNDERS, 2017.

[2] A. Katayev, C. Balciza, and D. W. Seccombe, "Establishing reference intervals for clinical laboratory test results: is there a better way?," American Journal of Clinical Pathology, vol. 133, no. 2, pp. 180-186, 2010.

[3] B. E. Statland and P. Winkel, "Effects of preanalytical factors on the intraindividual variation of analytes in the blood of healthy subjects: consideration of preparation of the subject and time of venipuncture," CRC Critical Reviews in Clinical Laboratory Sciences, vol. 8, no. 2, pp. 105-144, 1977.

[4] Clinical and Laboratory Standards Institute (NCCLS), Procedures for the Collection of Diagnostic Blood Specimens by Venipuncture; Approved Standard, NCCLS document H3-A5 (ISBN 1-56238-515-1). NCCLS, 940 West Valley Road, Suite 1400, Wayne, Pennsylvania 19087-1898 USA, Fifth edition, 2003.

[5] F. H. Wians, "Clinical laboratory tests: which, why, and what do the results mean?," Laboratoriums Medizin, vol. 40, no. 2, pp. 105-113, 2009. 
[6] L. Quintó, J. J. Aponte, J. Sacarlal et al., "Haematological and biochemical indices in young African children: in search of reference intervals," Tropical Medicine \& International Health, vol. 11, no. 11, pp. 1741-1748, 2006.

[7] X. Zhang, Y. Ding, Y. Zhang, J. Xing, Y. Dai, and E. Yuan, "Age- and sex-specific reference intervals for hematologic analytes in Chinese children," International Journal of Laboratory Hematology, vol. 41, no. 3, pp. 331-337, 2018.

[8] E. B. Robins and S. Blum, "Hematologic reference values for African American children and adolescents," American Journal of Hematology, vol. 82, no. 7, pp. 611-614, 2007.

[9] R. S. Kibaya, C. T. Bautista, F. K. Sawe et al., "Reference ranges for the clinical laboratory derived from a rural population in Kericho, Kenya," PLoS One, vol. 3, no. 10, article e3327, 2008.

[10] T. Miri-Dashe, S. Osawe, M. Tokdung et al., "Comprehensive reference ranges for hematology and clinical chemistry laboratory parameters derived from normal Nigerian adults," PLoS One, vol. 9, no. 5, article e93919, 2014.

[11] E. Saathoff, P. Schneider, V. Kleinfeldt et al., "Laboratory reference values for healthy adults from southern Tanzania," Tropical Medicine \& International Health, vol. 13, no. 5, pp. 612625, 2008.

[12] A. Al-Mawali, A. D. Pinto, R. Al-Busaidi, R. H. Al-Lawati, and M. Morsi, "Comprehensive haematological indices reference intervals for a healthy Omani population: first comprehensive study in Gulf Cooperation Council (GCC) and Middle Eastern countries based on age, gender and $\mathrm{ABO}$ blood group comparison," PLoS One, vol. 13, no. 4, article e0194497, 2018.

[13] NCCLS, How to Define and Determine Reference Intervals in the Clinical Laboratory; Approved Guideline-Second Edition. NCCLS Document C28-A2 (ISBN 1-56238-406-6), NCCLS, 940 West Valley Road, Suite 1400, Wayne, Pennsylvania 19087-1898, USA, 2000.

[14] T. Malati, "Whether western normative laboratory values used for clinical diagnosis are applicable to Indian population? An overview on reference interval," Indian Journal of Clinical Biochemistry, vol. 24, no. 2, pp. 111-122, 2009.

[15] W. Stevens, "Good clinical laboratory practice (GCLP): the need for a hybrid of good laboratory practice and good clinical practice guidelines/standards for medical testing laboratories conducting clinical trials in developing countries," Quality Assurance, vol. 10, no. 2, pp. 83-89, 2011.

[16] World Health Organization and Special Programme for Research and Training in Tropical Diseases, Good Clinical Laboratory Practice (GCLP), World Health Organization, Geneva, Switzerland, 2009.

[17] W. J. Dixon, "Processing data for outliers," Biometrics, vol. 9, no. 1, p. $74,1953$.

[18] S. Charache, A. Joffe, and H. Wong, "Hematologic changes during sexual maturation: why do black adolescents have low hemoglobin concentrations?," Journal of Adolescent Health Care, vol. 8, no. 4, pp. 315-321, 1987.

[19] W. A. Daniel, "Hematocrit: maturity relationship in adolescence," Pediatrics, vol. 52, no. 3, pp. 388-394, 1973.

[20] A. M. Buchanan, F. J. Muro, J. Gratz et al., "Establishment of haematological and immunological reference values for healthy Tanzanian children in Kilimanjaro Region," Tropical Medicine \& International Health, 2010.
[21] D. K. Dosoo, K. Kayan, D. Adu-Gyasi et al., "Haematological and biochemical reference values for healthy adults in the Middle Belt of Ghana," PLoS One, vol. 7, no. 4, article e36308, 2012.

[22] B. Kone, M. Maiga, B. Baya et al., "Establishing reference ranges of hematological parameters from Malian healthy adults," Journal of Blood \& Lymph, vol. 7, no. 1, 2017.

[23] N. Siraj, J. Issac, M. Anwar et al., "Establishment of hematological reference intervals for healthy adults in Asmara," BMC Research Notes, vol. 11, no. 1, p. 55, 2018.

[24] C. Zeh, P. N. Amornkul, S. Inzaule et al., "Population-based biochemistry, immunologic and hematological reference values for adolescents and young adults in a rural population in Western Kenya," PLoS One, vol. 6, no. 6, article e21040, 2011.

[25] N. Tembe, O. Joaquim, E. Alfai et al., "Reference values for clinical laboratory parameters in young adults in Maputo, Mozambique," PLoS One, vol. 9, no. 5, article e97391, 2014.

[26] J. Li, H. Zhang, X. Huang, J. Zhang, and X. Wu, "Establishment of reference intervals for complete blood count parameters in venous blood for children in the Xiamen area, China," International Journal of Laboratory Hematology, vol. 41, no. 5, pp. 691-696, 2019.

[27] J. M. Jorgensen, M. Crespo-Bellido, and K. G. Dewey, "Variation in hemoglobin across the life cycle and between males and females," Annals of the New York Academy of Sciences, vol. 1450, pp. 105-125, 2019.

[28] D. A. Gidlow, J. F. Church, and B. E. Clayton, "Seasonal variations in haematological and biochemical parameters," Annals of Clinical Biochemistry, vol. 23, no. 3, pp. 310-316, 1986.

[29] M. A. Sebok, E. P. Notari, L. A. Chambers, R. J. Benjamin, and A. F. Eder, "Seasonal temperature variation and the rate of donor deferral for low hematocrit in the American Red Cross," Transfusion, vol. 47, no. 5, pp. 890-894, 2007.

[30] J. Pluncevic Gligoroska, S. Gontarev, B. Dejanova, L. Todorovska, D. Shukova Stojmanova, and S. Manchevska, "Red blood cell variables in children and adolescents regarding the age and sex," Iranian Journal of Public Health, 2019.

[31] C. M. Hightower, "Seasonal hematocrit variation and health risks in the adult population of Kinshasa, Democratic Republic of Congo," Vascular Health and Risk Management, no. article 1001, 2009.

[32] M. Hu, T. Finni, M. Sedliak, W. Zhou, M. Alen, and S. Cheng, "Seasonal variation of red blood cell variables in physically inactive men: effects of strength training," International Journal of Sports Medicine, vol. 29, no. 7, pp. 564-568, 2008.

[33] E. Ruzagira, A. Abaasa, E. Karita et al., "Effect of seasonal variation on adult clinical laboratory parameters in Rwanda, Zambia, and Uganda: implications for HIV biomedical prevention trials," PLoS One, vol. 9, no. 8, article e105089, 2014.

[34] J. Okebe, J. Mwesigwa, S. C. Agbla et al., "Seasonal variation in haematological and biochemical reference values for healthy young children in the Gambia," BMC Pediatrics, vol. 16, no. 1, p. 5, 2016. 